\title{
Moderate Exercise Based on Artificial Gravity Preserves Orthostatic Tolerance and Exercise Capacity During Short-Term Head-Down Bed Rest
}

\author{
Xiao-Tao LI ${ }^{1,2 *}$, Chang-Bin YANG ${ }^{1 *}$, Yong-Sheng $\mathrm{ZHU}^{3 *}$, Jing SUN ${ }^{1}$, Fei SHI ${ }^{1}$, \\ Yong-Chun WANG ${ }^{1}$, Yuan GAO ${ }^{1}$, Jiang-Dong $\mathrm{ZHAO}^{\mathbf{1}}$, Xi-Qing $\mathrm{SUN}^{\mathbf{1}}$ \\ *These authors contributed equally to this work.
}

${ }^{1}$ Department of Aerospace Biodynamics, Faculty of Aerospace Medicine, Fourth Military Medical University, Xi'an, China, ${ }^{2}$ Department of Physical Education and Exercise Science, Xidian University, Xi'an, China, ${ }^{3}$ Department of Ultrasound Diagnosis, Xijing Hospital, Fourth Military Medical University, Xi'an, China

Received September 24, 2016

Accepted January 18, 2017

On-line April 12, 2017

\section{Summary}

Numerous countermeasures have been proposed to minimize microgravity-induced physical deconditioning, but their benefits are limited. The present study aimed to investigate whether personalized aerobic exercise based on artificial gravity (AG) mitigates multisystem physical deconditioning. Fourteen men were assigned to the control group $(n=6)$ and the countermeasure group $(\mathrm{CM}, \mathrm{n}=8)$. Subjects in the $\mathrm{CM}$ group were exposed to AG ( $2 \mathrm{Gz}$ at foot level) for $30 \mathrm{~min}$ twice daily, during which time cycling exercise of $80-95 \%$ anaerobic threshold (AT) intensity was undertaken. Orthostatic tolerance (OT), exercise tests, and blood assays were determined before and after 4 days head-down bed rest (HDBR). Cardiac systolic function was measured every day. After HDBR, OT decreased to $50.9 \%$ and $77.5 \%$ of pre-HDBR values in control and CM groups, respectively. Exercise endurance, maximal oxygen consumption, and AT decreased to $96.5 \%, 91.5 \%$ and $91.8 \%$ of pre-HDBR values, respectively, in the control group. Nevertheless, there were slight changes in the CM group. HDBR increased heart rate, sympathetic activity, and the pre-ejection period, but decreased plasma volume, parasympathetic activity and left-ventricular ejection time in the control group, whereas these effects were eliminated in the CM group. Aldosterone had no change in the control group but increased significantly in the CM group. Our study shows that 80-95\% AT aerobic exercise based on $2 \mathrm{Gz}$ of AG preserves OT and exercise endurance, and affects body fluid regulation during short-term HDBR. The underlying mechanisms might involve maintained cardiac systolic function, preserved plasma volume, and improved sympathetic responses to orthostatic stress.

\section{Key words}

Anaerobic threshold - Cardiovascular function - Heart rate variability • Maximal oxygen consumption • Microgravity • Short arm centrifuge

\section{Corresponding author}

X.-Q. Sun, Department of Aerospace Biodynamics, Faculty of Aerospace Medicine, Fourth Military Medical University, 17 Chang-Le-Xi Road, Xi'an, Shaanxi, China. Tel./fax: +86-2984774801. E-mail: sunxiqing@fmmu.edu.cn

\section{Introduction}

Physiological deconditioning is a consequence of manned space flight and ground-based simulated microgravity. This deconditioning comprises an impairment of cardiovascular function, which is represented by decreases in orthostatic tolerance (OT) and upright physical performance (Hughson 2009, Pavy-Le Traon et al. 2007). Orthostatic intolerance and reduced physical performance may be harmful to astronauts during reentry and after space flight. In particular, the ability to exit a landed spacecraft unaided 
during an emergency may be limited (Lee et al. 2010). Therefore, preservation of OT and physical capability during space flight is essential for the crew members to guarantee their safety and health.

In the past decades, numerous countermeasures, including physical exercise, wearing of load suits, lower body negative pressure (LBNP), gradient compression garments, saline loading, vibration, and pharmacological administration, have been proposed to counteract this physical deconditioning (Stenger et al. 2014, Hargens et al. 2012). However, most of these measurements achieved limited benefits. Exercise, as an effective indispensable method to maintain cardiovascular function, muscle metabolic, and neuromotor responses is prescribed to maintain or increase physical capacity in a normal-gravity environment. However, during space flight, the effectiveness of aerobic and resistive exercise countermeasures is not clear. Crew members on the international space station who perform treadmill running or cycling and moderate-intensity resistive exercises, still have considerable calf atrophy, loss of strength, and muscle morphological changes (Hargens et al. 2012). Regardless of these changes, aerobic exercise is still a critical countermeasure for prolonged space flight. Resistive and aerobic exercise, being considered as effective countermeasures to microgravity-induced muscle atrophy and a decrease in physical endurance, have become favorite countermeasures for astronauts (Pavy-Le Traon et al. 2007, Mulder et al. 2009, Trudel et al. 2012). However, neither aerobic nor resistive exercises can completely prevent the impairment in orthostatic tolerance after simulated or real microgravity (Guinet et al. 2009, Smith et al. 2008). Artificial gravity (AG) has been suggested as a universal countermeasure against deconditioning during microgravity because it acts almost on all physiological systems simultaneously. Ground-based studies have shown that AG produced by a short-arm centrifuge (SAC) increases OT in ambulatory men and prevents changes in cardiovascular function during microgravity (Linnarsson et al. 2015, Evans et al. 2004, Hargens et al. 2012, Stenger et al. 2012). However, most studies suggest that $A G$ alone is not sufficient for improving multisystem hypofunction during microgravity, and fails to maintain maximal oxygen consumption $\left(V \mathrm{O}_{2 \max }\right)$, heart rate (HR), or pulmonary ventilation during exercise (Greenleaf et al. 1999, Hargens et al. 2013).

To overcome these disadvantages, our previous studies focused on the combination of $\mathrm{AG}$ and exercise using a self-developed SAC with affiliated cycle ergometric exercise equipment. A series of HDBR experiments indicated that mild continuous aerobic exercise in alternating small doses of AG could attenuate changes in cardiac function and autonomic dysfunction, and increase leg venous flow resistance, but failed to prevent orthostatic intolerance (Wang et al. 2011, Yang et al. 2010, Yang et al. 2011, Yao et al. 2012). In our previous protocol, we set $40 \mathrm{~W}$ as the exercise intensity for every individual, and 1-2 intervals Gz as the AG dose.

However, after those experiments, two vital questions arose. One question was whether $40 \mathrm{~W}$ is sufficient to induce an apparent effect on cardiovascular function and the other question is whether a constant $40 \mathrm{~W}$ workload is suitable for each individual. The first question was answered in our previous studies (Guo et al. 2013, Wang et al. 2011, Yang et al. 2011, Yao et al. 2012), while a personalized workload may be the answer to the second question.

Therefore, in the present study, we set the power according to anaerobic threshold (AT), and attempted to determine the proper personalized cycling exercise intensity. We hypothesized that daily, alternating aerobic exercise for $1 \mathrm{~h}$ at an intensity of 80-95\% AT combined with $2 \mathrm{Gz}$ of $\mathrm{AG}$ produced by an $\mathrm{SAC}$ is able to prevent multisystem deconditioning associated with ground-based simulation of microgravity and then maintain OT and exercise endurance at the same time.

\section{Methods}

\section{Subjects}

Fourteen healthy men volunteered for this study. The present study conformed to the standards set by the latest version of the Declaration of Helsinki. The protocol was approved by the Ethics Committee for Human Research of the Fourth Military Medical University (No. LL-2013163-2). Each subject received verbal and written explanation of all experimental procedures before signing written informed consent and was aware of his right to withdraw from the experiment without prejudice at any time. All of the subjects passed medical and psychological screening examinations. The select examination included a detailed medical history, a physical examination, an electrocardiogram (ECG), an abdominalechography, a 20-min head-up tilt test without lower body negative pressure (intolerant subjects were excluded), $V \mathrm{O}_{2 \max }$ and AT measurements, as well as laboratory tests (hematology and blood chemistry). All of 
the subjects were no smoker, and had a regular lifestyle and were instructed to refrain from consuming alcohol, caffeinated beverages, and engaging in vigorous exercise for $24 \mathrm{~h}$ prior to testing and throughout the experiment. Subjects were randomly divided into two groups. 1) The control group ( $\mathrm{n}=6$; age: $20-26$ years; height: $169-174 \mathrm{~cm}$; body weight: $55-67 \mathrm{~kg}$ ) stayed 4 days in head-down bed rest (HDBR) without any countermeasures. 2) The countermeasure group (CM; $\mathrm{n}=8$; age: $20-28$ years; height: $168-176 \mathrm{~cm}$; weight: 53-70 kg) performed AG-based exercise during HDBR.

\section{Experimental design and conditions}

Subjects were housed in the Laboratory Center of Aerospace Medicine of the Fourth Military Medical University, China. The data collection room, bed rest room, and SAC training room are all on the same floor. All the room temperatures were maintained at approximately $24-27^{\circ} \mathrm{C}$. The experimental protocol included 2 days of an ambulatory control period, followed by 4 days of $-6^{\circ} \mathrm{HDBR}$, and then 2 days of a post-HDBR control period for testing and rehabilitation. The baseline data were collected in ambulatory control period, during which the subjects could familiarize themselves with the circumstances, eating food in bed, and experimental requirements. Subjects were housed in pairs and both members of the pair were in the same experimental group and performed each experiment or countermeasure at the same time. Before baseline (pre-HDBR) data collection, subjects experienced all of the testing procedures to become fully familiarized with the study. All of the testing was performed at the same time of day pre- and post-HDBR for each subject, and was at least $2 \mathrm{~h}$ postprandial.

During the course of HDBR, subjects strictly remained at $-6^{\circ}$ HDBR continuously for all daily activities, except for defecation. Subjects were monitored by video to ensure their compliance with the head-down tilt position. During the whole period of the experiment, dietary intake was $2300-2500 \mathrm{kcal} / \mathrm{day}$, and water intake was 1.0-1.5 $\mathrm{l} /$ day according to body weight and Chinese flight nutritional requirements (carbohydrate, fat and protein ratio was 55:30:15). Caffeine, cocoa, chocolate, tea, and herbal beverages were not allowed. Body weight, HR, systolic blood pressure (SBP), diastolic blood pressure (DBP), and mean arterial blood pressure (MBP) were measured in the morning before breakfast. During HDBR, subjects in the CM group received 30 min of AG and aerobic exercise twice daily as a countermeasure, while subjects in the control group remained HDBR position.

\section{Exercise tests}

The exercise test was performed 2 days before HDBR, which served as the baseline, and was then repeated in the course of post-HDBR after all other cardiovascular and OT tests were conducted. Upright aerobic power, or $V \mathrm{O}_{2 \max }$, was measured by an upright, graded cycling exercise test protocol before and after HDBR. Subjects cycled on a weight-braked cycling ergometer (894E, Monark Exercise AB, Varberg, Sweden). The protocol was modified according to a previous report (Moore et al. 2010, Stenger et al. 2012). In short, the test began with 5-min stages at work power of $50 \mathrm{~W}$, and then the work rate was increased every 2 min at $25 \mathrm{~W}$ increments until the subject could no longer maintain the desired cycling cadence $(60 \mathrm{rpm})$ or was unable to continue. HR and rhythm were continuously monitored by the standard II limb lead using an ECG module of PowerLab8/30 data acquisition system (ADInstruments, Sydney, Australia) and BP was monitored by a beat-by-beat photoplethysmography device (Finometer PRO, Finapres Medical Systems B.V., Arnhem, The Netherlands) during every test. Subjects breathed through a respiratory mask, which was connected to a metabolic gas analysis system (MetaMax 3B, CORTEX Biophysik, Leipzig, Germany) that was calibrated before each data collection session according to the reference manual. Measurements of HR, the volume of oxygen consumed per minute $\left(\mathrm{VO}_{2}\right)$, volume of carbon dioxide produced per minute $\left(V \mathrm{CO}_{2}\right)$, and respiratory exchange ratio (RER) were averaged in 20 -s samples throughout the graded exercise test. $V \mathrm{O}_{2 \max }$ was determined as the average of the highest level of $V \mathrm{O}_{2}$ measured in two consecutive $20 \mathrm{~s}$ samples and accepted when at least two of the following criteria were achieved: RER was greater than 1.1, peak HR was greater than $85 \%$ of the age-predicted maximum, a plateau of the $\mathrm{VO}_{2}$ curve was observed $(<150 \mathrm{ml} / \mathrm{min}$ increase) and/or the 60 -rpm pace could not be maintained and volitional fatigue was reached. Exercise endurance was expressed as duration time of the whole exercise (unit: $\min$ ). $V \mathrm{O}_{2 \max }$ and AT were achieved by MetaSoft3.0 (CORTEX Biophysik, Leipzig, Germany) after the test.

\section{Artificial gravity-based exercise countermeasure}

AG was produced by SACIII, a successor to our self-developed SAC (China patent No.200710018297.4), 
with a diameter of $4 \mathrm{~m}$, as reported in our previous study (Wang et al. 2011, Yao et al. 2012). The distance between the heart of the subject and the center of rotation was approximately $70 \mathrm{~cm}$ in the supine position. Subjects in the $\mathrm{CM}$ group performed cycling exercise during centrifuge-induced AG twice per day, and this exercise started at 09:30 $\mathrm{h}$ and 15:00 $\mathrm{h}$ during HDBR. In each session, the AG-based exercise consisted of $30 \mathrm{~min}$ constant $2.0 \mathrm{Gz}$ of AG at foot level and three cycles of aerobic exercise with exercise intensity ranging from $80-95 \%$ AT. All of the subjects kept the head still to prevent nausea or vertigo during the cycling and rotation.

\section{Evaluation of orthostatic tolerance}

Head-up tilt combined with the LBNP test was applied before and after HDBR to assess OT. Both tests were performed at the same time on the day of data collection. The study was performed in a quiet room and the temperature was maintained at $24-26^{\circ} \mathrm{C}$. Subjects were placed supine on an automated tilt table (Heping, Beijing, China). During the OT test, SBP and DBP was obtained beat-by-beat at the finger on the right hand by Finometer PRO. ECG was monitored by PowerLab. After being connected to the equipment, subjects remained at rest in the supine position for $15 \mathrm{~min}$ to collect baseline data. They were then tilted to $75^{\circ}$ head up for $30 \mathrm{~s}$, followed by increasing steps of LBNP. LBNP began at $-20 \mathrm{~mm} \mathrm{Hg}$ for $5 \mathrm{~min}$, followed by an increase of $-20 \mathrm{~mm} \mathrm{Hg}$ every $5 \mathrm{~min}$ until $-60 \mathrm{~mm} \mathrm{Hg}$ (total duration is $20 \mathrm{~min}$ ) or presyncope occurred. The test was terminated immediately if one of the following signs occurred: a sudden decline in SBP $(>25 \mathrm{~mm} \mathrm{Hg} / \mathrm{min})$, a sudden decline in DBP $(>15 \mathrm{~mm} \mathrm{Hg} / \mathrm{min})$; a sudden decline in HR ( $>15$ beats/min); SBP bellow $70 \mathrm{~mm} \mathrm{Hg}$; and occurrence of nausea, dizziness, clammy skin, pallor; or any combination of the above. The duration time of negative pressure was used to evaluate OT (unit: $\mathrm{min}$ ).

\section{Spectral power analyses of heart-rate variability (HRV)}

HRV determined by the R-R variability series was analyzed by a HRV module of LabChart7.0 (ADInstruments, Sydney, Australia). ECG was obtained from the baseline data of OT test when subjects remained at rest in the supine position. To avoid the disturbing, spontaneous respiration of subjects was controlled to 15 times per minute during the measurement process. After the R-R interval obtained by an ECG signal was detected, fast Fourier transformation was applied to compute power spectral density. Low-frequency power of the R-R interval (LF) was defined in the range $0.04-0.15 \mathrm{~Hz}$. High-frequency power of R-R interval (HF) was defined in the range $0.15-0.40 \mathrm{~Hz}$. Normalized low-frequency power (LFn) and normalized high-frequency power (HFn) were calculated using the formula: $L F n=L F /(L F+H F) \times 100 \%$ and $H F n=H F /(L F+H F) \times 100 \%$, respectively.

\section{Blood assays}

Blood samples were collected at the same time on the morning before and after HDBR. All of the blood samples were analyzed in the clinical laboratory of Xijing Hospital. Aldosterone concentration levels in the plasma were assayed by ELISA kits (IBL International $\mathrm{GmbH}$, Hamburg, Germany) according to the test protocol of the manufacturer, and were analyzed on a Fluostar OPTIMA Microplate Reader (BMG Labtech, Ortenberg, Germany). The percent change in plasma volume $(\% \Delta \mathrm{PV})$ was calculated using the formula of van Beaumont (1972): $\% \Delta P V=100 /\left(100-H c t_{1}\right) \times\left(H_{c t}-H_{1}-t_{2}\right) / H_{c t} \times 100 \%$. Where Hct is the hematocrit value measured from blood samples.

\section{Measurement of cardiac systolic function}

Cardiac systolic function was measured daily in the morning. After ECG leads being connected, a pressure sensor connected to the cardiac function detecting device (Zhenqin, Xi'an, China) was fixed upon the carotid artery by a fixing apparatus. Continuous carotid pulse, phonocardiogram and ECG were simultaneously recorded. Cardiac systolic function, including left ventricular ejection time (LVET), pre-ejection period (PEP), and ejection fraction (EF) were calculated and reported by this device. These parameters were calculated automatically through averaging five normal shapes of waves. Each subject had been trained to breath in synchrony with an electronic metronome at a rate of $15 / \mathrm{min}$ to avoid hypo or hyper-ventilation during the experiments.

\section{Statistical analysis}

All of the values are expressed as mean \pm SD. The outcomes of cardiac systolic function between the groups were compared by repeated measures analysis of variance (ANOVA). If a significant effect was detected, the ANOVA test for comparisons of means between the two groups was used to determine which specific values were significantly different. The differences in values of pre-, post-, and during HDBR were examined by one-way 
Table 1. Body weight, BP, and HR before, during, and after head-down bed rest in both Con and CM groups.

\begin{tabular}{lccccccc}
\hline & & Pre-HDBR & Day 1 & Day 2 & Day 3 & Day 4 & Post-HDBR \\
\hline \multirow{3}{*}{$B W(\mathrm{~kg})$} & $C o n$ & $66.8 \pm 8.5$ & $66.7 \pm 9.4$ & $66.8 \pm 8.8$ & $66.9 \pm 9.5$ & $66.9 \pm 9.2$ & $66.5 \pm 9.1$ \\
& $C M$ & $64.6 \pm 6.6$ & $64.6 \pm 7.6$ & $64.5 \pm 6.7$ & $64.9 \pm 7.2$ & $64.7 \pm 7.9$ & $64.8 \pm 7.2$ \\
$\mathrm{HR}(\mathrm{bpm})$ & $C o n$ & $67.1 \pm 11.6$ & $63.1 \pm 8.3^{*}$ & $67.1 \pm 7.6$ & $67.1 \pm 9.6$ & $67.1 \pm 8.6$ & $71.6 \pm 9.2^{*}$ \\
& $C M$ & $62.9 \pm 3.4$ & $60.9 \pm 5.4^{*}$ & $62.9 \pm 6.8$ & $63.2 \pm 8.5$ & $64.9 \pm 7.8$ & $65.7 \pm 6.6$ \\
$\mathrm{SBP}(\mathrm{mm} \mathrm{Hg})$ & $C o n$ & $120.1 \pm 9.3$ & $123.0 \pm 11.3$ & $120.1 \pm 9.2$ & $119.5 \pm 8.4$ & $120.0 \pm 10.3$ & $119.7 \pm 12.6$ \\
& $C M$ & $118.8 \pm 8.8$ & $120.9 \pm 8.8$ & $119.4 \pm 7.2$ & $119.8 \pm 6.5$ & $119.4 \pm 10.8$ & $120.3 \pm 11.5$ \\
\multirow{2}{*}{$\mathrm{BP}(\mathrm{mm} \mathrm{Hg})$} & $C o n$ & $72.3 \pm 8.6$ & $73.3 \pm 6.7$ & $71.6 \pm 7.5$ & $72.4 \pm 5.2$ & $71.6 \pm 4.9$ & $73.4 \pm 10.1$ \\
\multirow{3}{*}{$\mathrm{MAP}(\mathrm{mm} \mathrm{Hg})$} & $C M$ & $71.9 \pm 9.2$ & $71.9 \pm 9.2$ & $71.9 \pm 9.2$ & $71.9 \pm 9.2$ & $71.9 \pm 9.2$ & $72.3 \pm 11.4$ \\
& $C o n$ & $88.2 \pm 9.1$ & $89.9 \pm 8.2$ & $87.8 \pm 8.1$ & $88.1 \pm 6.3$ & $87.7 \pm 6.7$ & $88.8 \pm 10.9$ \\
& $C M$ & $87.6 \pm 9.1$ & $88.3 \pm 8.1$ & $87.7 \pm 7.5$ & $87.9 \pm 8.3$ & $87.7 \pm 9.7$ & $88.3 \pm 11.4$ \\
\hline
\end{tabular}

Data are expressed as mean $\pm \mathrm{SD}$. $* P<0.05$, post-HDBR vs. pre-HDBR. As compared with pre-HDBR, HR decreased significantly in both groups in the first day of HDBR, and increased significantly in the Con group after HDBR. No significant changes in body weight, SBP, DBP, and MBP were detected in either group throughout HDBR. HDBR, head-down bed rest; Con, control group; $\mathrm{CM}$, countermeasure group; BW, body weight; HR, heart rate; SBP, systolic blood pressure; DBP, diastolic blood pressure; MAP, mean arterial pressure.

ANOVA. The data of body weight, HR, BP, OT, the exercise test, $\mathrm{HRV}, \mathrm{PV}$, and aldosterone concentration levels in the plasma were analyzed by the Student's t-test. Significance was accepted when $P<0.05$.

\section{Results}

Body weight, arterial blood pressure, and heart rate

Mean values of body weight, SBP, DBP, MBP, and HR are shown in Table 1. As compared with pre-HDBR, HR decreased significantly in both groups on day-1 of HDBR, which increased significantly after HDBR in the control group $(P<0.05)$. No significant changes in body weight, SBP, DBP, or MBP were detected in either group throughout HDBR.

\section{Orthostatic tolerance}

OT data before and after HDBR are shown in Figure 1. After HDBR, the time of OT in the control group decreased from $16.13 \pm 3.36$ to $8.21 \pm 1.74 \mathrm{~min}(P<0.05)$, which was $50.9 \%$ of pre-HDBR values, whereas the time of OT in the CM group decreased from $14.56 \pm 5.31 \mathrm{~min}$ to $11.29 \pm 2.19 \mathrm{~min}$, which was $77.5 \%$ of pre-HDBR values. The time of OT after HDBR was significantly lower in the control group compared with the $\mathrm{CM}$ group (8.21 $\pm 1.74 \mathrm{~min}$ vs. $11.29 \pm 2.19 \mathrm{~min}$, respectively, $P<0.05)$.

\section{Exercise capacity}

Exercise test data before and after HDBR are shown in Figure 2. In the control group, exercise

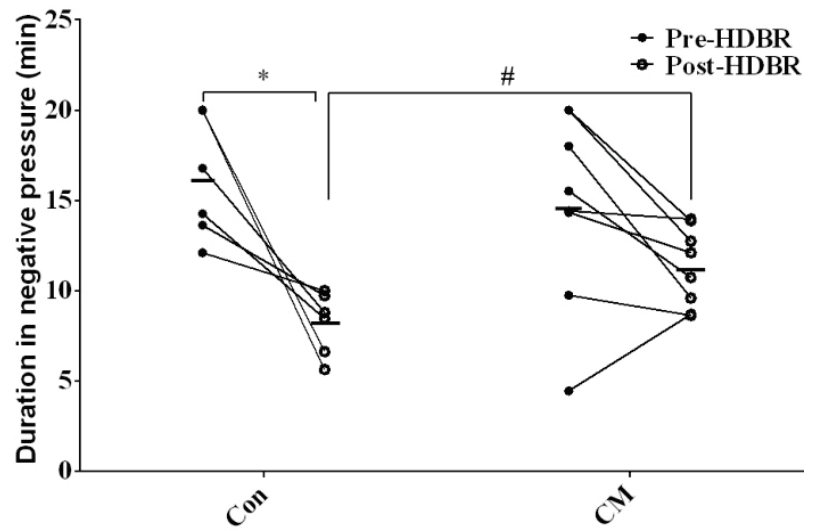

Fig. 1. Duration time of OT before and after HDBR in both Con and $\mathrm{CM}$ groups. As compared with pre-HDBR, OT decreased significantly in Con group, while no remarkable changes were observed in $\mathrm{CM}$ group. ${ }^{*} P<0.05$, post-HDBR vs. pre-HDBR in Con group. \# $P<0.05$, CM group vs. Con group post-HDBR. The horizontal lines represent as mean values. Con, control group; $\mathrm{CM}$, countermeasure group.

endurance decreased from $14.09 \pm 2.42 \mathrm{~min}$ to $13.59 \pm$ $2.26 \min (P<0.05)$, which was $96.5 \%$ of pre-HDBR values. $V \mathrm{O}_{2 \max }$ decreased from $31.84 \pm 2.92 \mathrm{ml} \cdot \mathrm{min}^{-1} \cdot \mathrm{kg}^{-1}$ to $29.18 \pm 3.85 \mathrm{ml} \cdot \mathrm{min}^{-1} \cdot \mathrm{kg}^{-1}(P<0.05)$, which was $91.5 \%$ of pre-HDBR values. AT decreased from $23.51 \pm$ $9.29 \mathrm{ml} \cdot \mathrm{min}^{-1} \cdot \mathrm{kg}^{-1}$ to $21.59 \pm 8.54 \mathrm{ml} \cdot \mathrm{min}^{-1} \cdot \mathrm{kg}^{-1}(P<0.01)$, which was $91.8 \%$ of pre-HDBR values. However, exercise endurance, $V \mathrm{O}_{2 \max }$, and $\mathrm{AT}$ did not change appreciably in the CM group compared with pre-HDBR. Mean time of exercise endurance in the CM group seems not changed. There were significant differences between control and $\mathrm{CM}$ groups with respect to $V \mathrm{O}_{2 \max }$ 
$\left(29.18 \pm 3.85 \mathrm{ml} \cdot \mathrm{min}^{-1} \cdot \mathrm{kg}^{-1}\right.$ vs. $35.36 \pm 5.54 \mathrm{ml} \cdot \mathrm{min}^{-1} \cdot \mathrm{kg}^{-1}$, $P<0.05)$, and AT $\left(21.59 \pm 8.54 \mathrm{ml} \cdot \mathrm{min}^{-1} \cdot \mathrm{kg}^{-1}\right.$ vs. $26.3 \pm$ $\left.4.2 \mathrm{ml} \cdot \mathrm{min}^{-1} \cdot \mathrm{kg}^{-1}, P<0.05\right)$, respectively after HDBR.
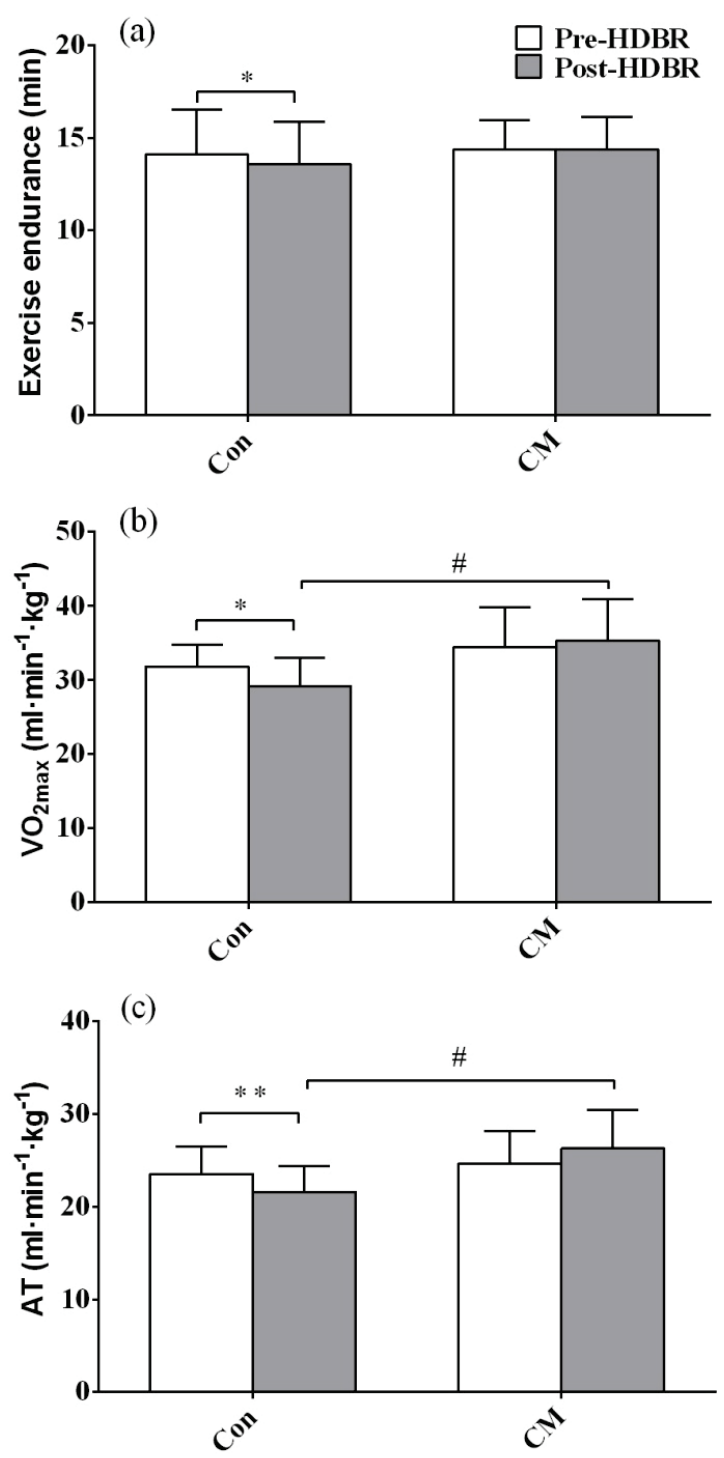

Fig. 2. The exercise endurance, $V_{2 \max }$ and $A T$ before and after HDBR. (a) Mean value of exercise endurance, (b) mean value of maximal oxygen uptake $\left(V_{2 \max }\right),(\mathrm{c})$ mean value of anaerobic threshold (AT). As compared with pre-HDBR, exercise endurance, $\mathrm{V}_{2 \max }$, and AT decreased signifcantly in Con group, while no reamarkble changes were oberved in $\mathrm{CM}$ group. $* P<0.05$ and $* * P<0.01$, post-HDBR vs. pre-HDBR in the control group. $\# P<0.05$, the countermeasure group vs. the control group at post-HDBR. Con, control group; CM, countermeasure group.

\section{Cardiac systolic function}

Data of cardiac systolic function before, during, and after HDBR are shown in Figure 3. During HDBR, some distinct changes between control and CM groups were observed. In the control group, LVET and EF tended to decline, and compared with pre-HDBR the value of LVET became significant lower on day-3
$(P<0.05)$, the value of EF was significantly lower on day-4 $(P<0.05)$. In the $\mathrm{CM}$ group, the $\mathrm{EF}$ value did not change, and LVET tended to increase and reached significance on day-3 $(P<0.05)$ and day-4 $(P<0.05)$ compared with that in control group. In the control group, compared with pre-HDBR, PEP and PEP/LVET tended to increase, and PEP value became significantly higher on day-3 $(P<0.05)$ and day-4 $(P<0.05)$. The PEP/LVET value reached significance on day-3 $(P<0.05)$. In the CM group, compared with pre-HDBR, values of PEP and PEP/LVET tended to decline, PEP reached significance on day-4. As compared with control group PEP/LVET became significant lower on day-3, day-4 and post-HBR.

\section{Heat rate variability}

HRV data before and after HDBR are shown in Figure 4. In the control group, HFn decreased from $61.52 \pm 6.74 \%$ to $45.22 \pm 13.76 \%(P<0.01)$, LFn increased from $26.51 \pm 9.58 \%$ to $44.36 \pm 15.54 \% \quad(P<0.05)$, and LF/HF increased from $0.44 \pm 0.17$ to $1.08 \pm 0.61(P<0.05)$. No significant changes in HFn, LFn, or LF/HF were observed in the CM group.

\section{Plasma volume and aldosterone}

After HDBR, PV decreased significantly by $9.15 \pm 6.5 \%$ in the control group, while decreased by $0.78 \pm 1.97 \%$ in the $\mathrm{CM}$ group. There is significant difference between the groups $(\mathrm{P}<0.05)$. Aldosterone levels were not changed after HDBR compared with pre-HDBR $(132.85 \pm 12.60 \mathrm{pg} / \mathrm{ml}$ vs. $147.67 \pm 49.95 \mathrm{pg} / \mathrm{ml}$, $P=0.47)$ in the control group. However, in the CM group aldosterone levels were increased significantly by $28.6 \%$ compared with pre-HDBR $(133.75 \pm 16.80 \mathrm{pg} / \mathrm{ml}$ vs. $172.14 \pm 28.49 \mathrm{pg} / \mathrm{ml}, P<0.05)$.

\section{Discussion}

The protocol of artificial gravity combined with exercise as a countermeasure

Even though various countermeasures have been investigated, exercise and $A G$ exposure appear to be indispensable (Hargens et al. 2012). Previous studies (Convertino et al. 1982a, Lee et al. 2010) have suggested that an effective countermeasure to a real or simulated gravity should include some gravity component. Mulder et al. (2015) reported that a short and versatile daily exercise preserved muscle size and increased knee extensor isometric strength but failed to preserve muscle power and bone integrity. 

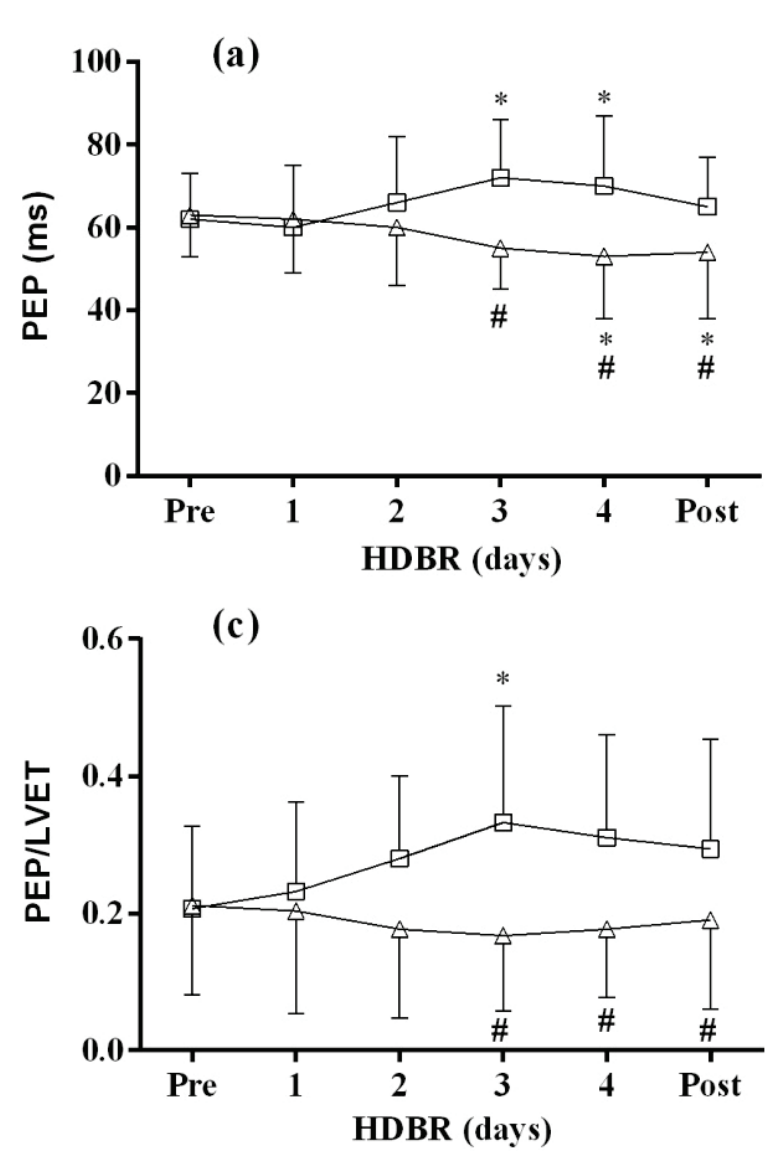

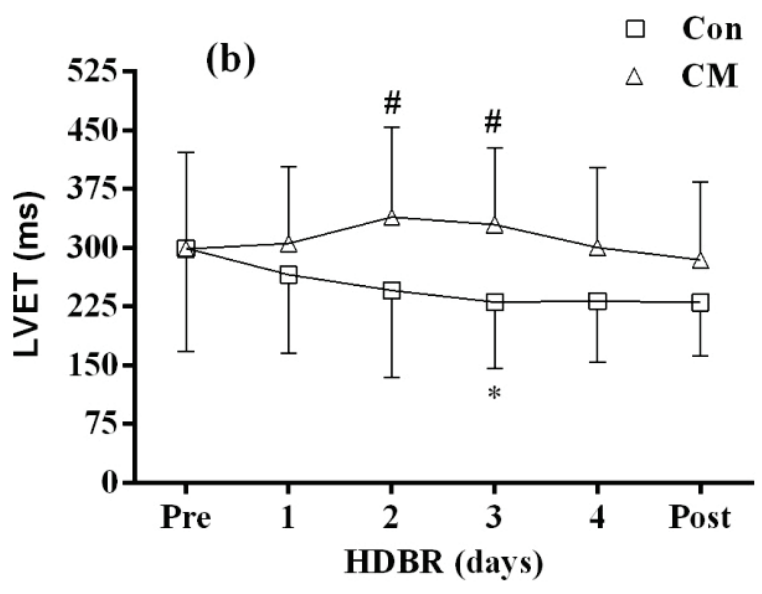

(d)

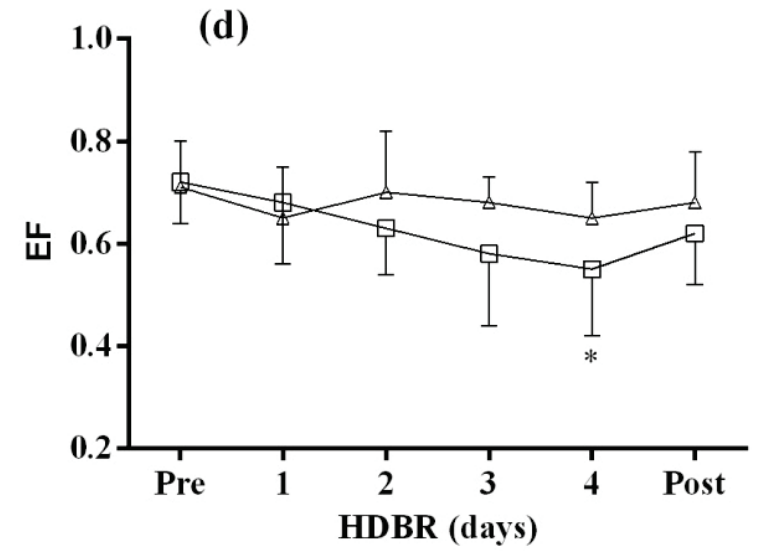

Fig. 3. Cardiac systolic function before, during and after HDBR in Con and CM groups. (a) Mean values of pre-ejectionperiod (PEP), (b) mean values of left ventricular ejection time (LVET), (c) mean values of PEP/LVET ration, (d) mean values of ejection fraction (EF). As compared with pre-HDBR, PEP and PEP/LVET increased significantly, while LVET and EF decreased significantly during HDBR in Con group. As compared with Con group, EF was unchanged, LVET was increased, PEP and PEP/LVET were decreased significantly during HDBR in CM group. As compared with pre-HDBR PEP decrease significanltly from 4th day to post-HDBR. $* P<0.05$, post-HDBR vs. pre-HDBR in control group. $\# \mathrm{P}<0.05$, the countermeasure group vs. the control group. Con, control group; CM, countermeasure group.

Iwasaki et al. (2001) reported that 1-h doses of $2 \mathrm{Gz}$ (at the foot level) AG exposure daily prevented the negative effects of HDBR on baroreflex function and PV. Other studies suggested that 1-h doses of $1.0 \mathrm{Gz}$ (at the heart level) AG exposure daily effectively attenuated HDBR-induced OT (Moore et al. 2010, Lee et al. 2010, Hargens et al. 2012, Evans et al. 2004). Linnarsson et al. (2015) reported that $30 \mathrm{~min}$ or six periods of $5 \mathrm{~min}$ with 3-min intervals AG ( $1 \mathrm{Gz}$ at the mess center, $2 \mathrm{Gz}$ at the foot) exposure daily effectively preserved OT and aerobic power after HDBR, but with limited benefits to PV. Rittweger et al. (2015) reported that AG preserved vertical jump performance, but failed to prevent HDBRinduced alteration in muscle and bone metabolism. Linnarsson and Rittweger advised future studies of AG should combined with exercise, and they claimed an intermittent protocol with six periods of 5 min AG was better tolerated than a protocol without breaks, and was also more effective. However, in our present study we find that a constant artificial gravity is caused less vestibular stimulation and could be accepted easily by ordinary individuals (the data is to be published in the future). Results from previous HDBR studies were inconsistent regarding $\mathrm{AG}$ alone as a countermeasure for decreased $V \mathrm{O}_{2 \max }$ countermeasures, such as quiet standing (Vernikos et al. 1996) and use of a reverse pressure gradient garment (Convertino et al. 1982b), provided full or partial protection against the decline in $V \mathrm{O}_{2 \max }$ in some HDBR studies.

Compared with these divergent results, AG combined with aerobic exercise produces a more consistent benefit for maintenance of exercise capacity. AG combined with cycle ergometric exercise was proposed as a countermeasure for microgravity-induced deconditioning. Katayama et al. (2004) demonstrated that AG combined with $60 \mathrm{~W}$ exercise was sufficient to retain upright exercise responses. Our previous study showed that $1 \mathrm{~h} \mathrm{AG}(2 \mathrm{Gz}$ at the foot level and approximately 
$0.7 \mathrm{Gz}$ at the heart level) exposure combined with $40 \mathrm{~W}$ cycling exercise daily provided more benefit than the same AG or exercise regimen alone in cardiovascular function and autonomic regulation (Yang et al. 2010). Further, this training program was applied in the short-term HDBR and the results were positive.
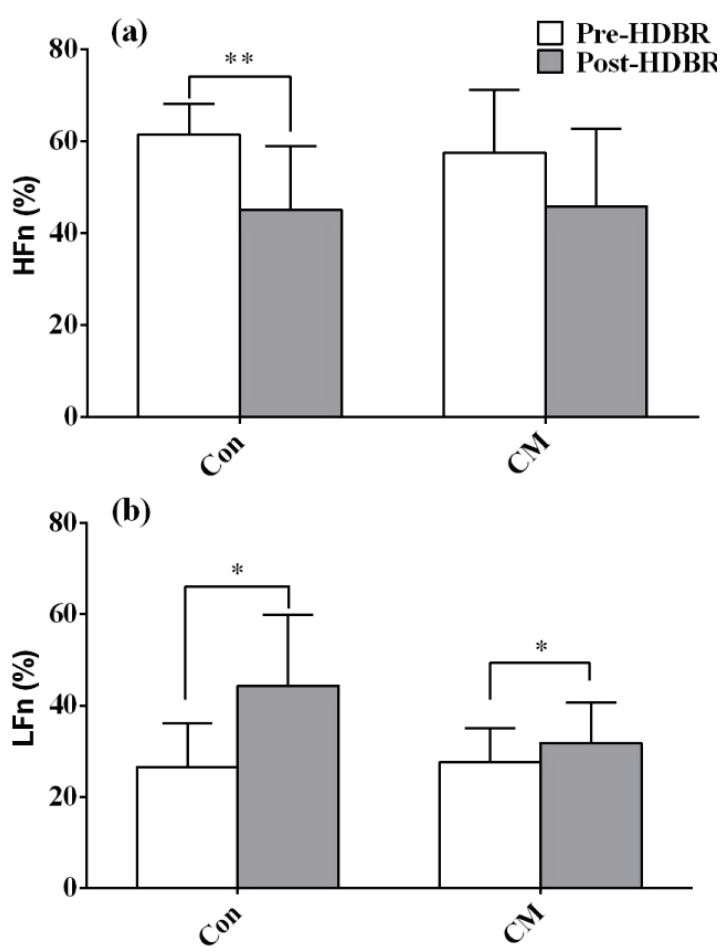

(c)

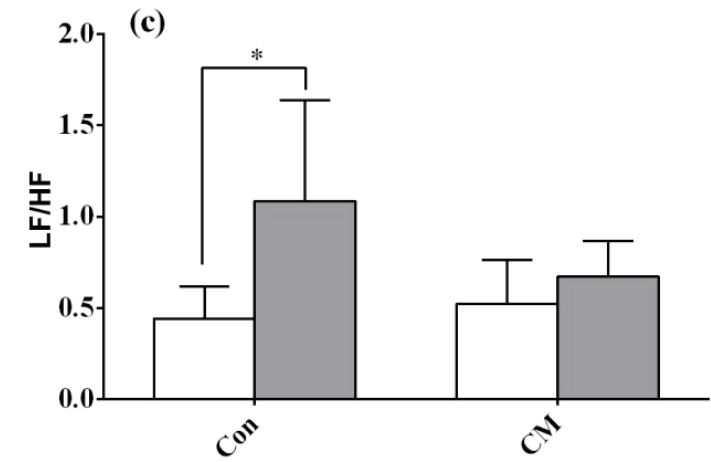

Fig. 4. Changes of HRV before and after HDBR in Con and CM groups. (a) Normalized high frequency power (HFn), (b) normalized low frequency power (LFn), (c) the ratio of low to high frequency power (LF/HF). As compared with pre-HDBR, HFn was increased signifcantly, LFn and LF/HF increased signifcantly in the control group, while no remarkable changes were observed in $\mathrm{CM}$ group. $* P<0.05, * * P<0.01$, post-HDBR vs. pre-HDBR. Con, control group; CM, countermeasure group; HRV, heart rate variability.

We found that constant exercise intensity was not suitable for everyone because of different physical fitness of each individual. In the present study, the minimal exercise load is more than $100 \mathrm{~W}$, which was depended on each individual's workload at the AT point. $40 \mathrm{~W}$ or even $60 \mathrm{~W}$ workload was really a mild intensity. In the present study, HR response to standing is almost equal to which response to $50 \mathrm{~W}$ workload (the data is to be published). In the studies of Katayama et al. (2004), Iwasaki et al. (2005), $60 \mathrm{~W}$ workload of exercise were prescribed, but two subjects failed to tolerate. This was because of the hypergravity $(1.2 \mathrm{Gz}$ at the heart, $3.5 \mathrm{Gz}$ at the foot level) instead of exercise load. Therefore, the exercise intensity should prescribed base on the $V \mathrm{O}_{2 \max }$ or AT. We choose percent of AT as unit of exercise workload, because this personalized exercise is aerobic metabolism dominated, and we had supposed that an aerobic exercise is enough to counter the HDBRinduced physical deconditioning.

Furthermore, the AG intensity in our previous studies was not appropriate enough. Goswami et al. (2015) reported that $2 \mathrm{Gz}$ of $\mathrm{AG}$ (at the foot level) exposure provides a similar cardiovascular and cerebral responses to standing, which is consistent with our finding in the preliminary experiments. Therefore, $2 \mathrm{Gz}$ of AG may be suited as a countermeasure for microgravity induced deconditioning of cardiovascular and cerebrovascular.

The present study proposed a practical and convenient AG-based exercise protocol, and the results demonstrated that AG ( $2 \mathrm{Gz}$ at the foot level) combined with aerobic cycling exercise (80-95\% AT) successfully prevented the HDBR-induced changes in OT, exercise capacity, cardiac systolic function, $\mathrm{HRV}, \mathrm{PV}, V \mathrm{O}_{2 \max }$, AT, sympathetic nervous tone, and aldosterone concentration levels.

\section{Cardiac systolic function}

In the present study, we found that PEP and PEP/LVET increased significantly, while LVET and EF decreased significantly during HDBR in the control group, which suggested that the cardiac systolic function was decreased by HDBR. The results were consistent with previous study (Yang et al. 2011). However, in the CM group, EF was unchanged, LVET was increased, PEP and PEP/LVET were decreased significantly during HDBR compared with the control group. This suggested that the cardiac systolic function was effectively preserved by the AG-based cycling exercise. During HDBR, the body fluid and blood were shifted immediately from the lower body to the upper body, the circulation volume increased. The increased returned blood volume induced the increase of ventricular filling. 
The ventricular filling also called pre-load. Appropriate initial length is conducive to muscle contraction. Therefore, if the ventricle is pre-stretched by a greater filling then the contraction force or tension generated during systole is increased and stroke volume and EF increases. This can be seen during the beginning of the HDBR, but all the state will changed soon, and the cardiovascular system would adapted to the new state soon, and the stroke volume and EF also returned to normal situation. As time went on, the PV decreased and the pre-load decreased to some extent. However, the returned blood was still more than normal. Besides, there was no need to overcome external load for standing, walking or anything else. Therefore the cardiac systolic faction was decreased by the HDBR as time went on. However, subjects in the CM group had to perform cycling exercise and exposed to AG. During exercise, the cardiac had to overcome the external workload, and the cardiac muscles were exercised. The exposing to AG strengthened this effect by providing a similar orthostatic stress, which was also helpful to preserve PV, and improve sympathetic response to stress of orthostatic and exercise. The improvement in cardiac systolic function may be connected with the fitness of each individual who participated AG-based exercise. All the subjects were ordinary students without athlete experience from college or graduate school. The exercise intensity is suitable for the improvement of their aerobic power. The reasons why AG-based exercise improves cardiac systolic function should be further explored. Such observations suggest that AG-based exercise treatment may help maintain OT and exercise capacity by preventing cardiac deconditioning during HDBR.

\section{Cardiac autonomic nervous regulation}

In the present study HFn was decreased, while LFn and LF/HF were increased in the control group. This is in accordance with previous studies (Wang et al. 2011, Stenger et al. 2012). These studies suggested that a relatively higher OT benefited from AG-based exercise is related to lower LF/HF. As a non-invasive tool for quantifying sympathetic and vagal modulation of $\mathrm{HR}$ in human studies, spectral analysis of HRV has been applied in HDBR and space flight. Previous reports have shown that HFn of the HRV is decreased in association with an increase in baseline HR, suggesting vagal withdrawal after HDBR (Cooke et al. 2000, Custaud et al. 2002, Iwasaki et al. 2005, Wang et al. 2011). The increased HR was found in the present study as well. Reductions in parasympathetic and increases in sympathetic after space flight have been confirmed by analysis of BP variability (Evans et al. 2004, Fritsch-Yelle et al. 1994, Waters et al. 2002). Evans et al. (2004) suggested that low frequency oscillations in BP, as a reliable noninvasive index of sympathetic nervous system activity (Pagani et al. 1986) was correlated with increased OT. LF/HF is an indicator of cardiac sympathovagal balance regulated by sympathetic or vagal nervous on the sinus node. The cardiac autonomic regulation is affected by respiration, cardiac mechanics, arterial compliance, baroreflex, and sinus node sensitivity (Brown et al. 1993, Hirsch and Bishop 1981, Iwasaki et al. 2001, Levine et al. 1997). With changes in arterial pressure variability as the input signal of the baroreflex system, HRV is affected by changes in cardiac mechanics or arterial compliance (Levine et al. 1997). Therefore, differences in cardiac systolic function between control and AG-based exercise treatment might contribute to the alterations of HRV. Autonomic dysregulation with alteration of the sympathetic-parasympathetic balance were a crucial factor in cardiovascular deconditioning.

\section{Plasma volume and aldosterone}

In the present study, PV decreased by $9.15 \%$ compared with pre-HDBR in the control group, which conformed to previous studies (Iwasaki et al. 2012, Linnarsson et al. 2015). The AG-based exercise preserved $\mathrm{PV}$ in the $\mathrm{CM}$ group effectively. Aldosterone levels were unchanged in the control group after HDBR, but they were increased in the CM group. During space flight and HDBR, blood and fluids were shifted immediately from the lower to the upper body (Norsk et al. 2014), which induces changes of hormones that regulate blood volume (Watenpaugh 2001). Maillet et al. (1994, 1995) studied the hormone changes induced by $37.5 \mathrm{~h} \mathrm{HDBR}$, they found that after $24 \mathrm{~h}$ of HDBR, rennin activity in plasma was increased $60 \%$, as a response to contraction of plasma volume, the reninangiotensin-aldosterone system was enhanced after HDBR due to fluid shift and adaptation in blood volume (Maillet et al. 1994, Maillet et al. 1995).

Stenger et al. (2012) found remarkable increase in aldosterone levels in the supine position in both control and AG treatment group after 21 days of HDBR, and it increased more in the upright position. Schmedtje et al. (1996) found that aldosterone might remain stable with a high average salt intake during HDBR. Other studies could explain these divergent results (Hinghofer-Szalkay 
et al. 2002a,b), where the authors examined blood volume regulating hormones with high and low sodium supply during HDBR, and found that resting hormone levels were not altered by HDBR, but aldosterone levels were lower with a high sodium intake than with a low sodium intake. These findings suggest an important potential underlying mechanism where hormonal activity is more affected by oral salt load than by HDBR. Therefore, the state of sodium intake during HDBR is a critical factor involved in aldosterone regulation. In our study, sodium intake was controlled, and a positive effect of AG-based aerobic exercise on an increase in aldosterone levels was observed. Stenger et al. (2012) reported that subjects who underwent daily, 1-h exposure to 1.0 Gz AG (at heart level) during HDBR had increased levels of norepinephrine in the upright position and preserved OT. In the present study, aldosterone was thought to be an indicator of activation of the sympathetic nervous system to orthostatic stress. Enhanced activity in the sympathetic nervous during orthostatic stress is correlated with increased OT (Evans et al. 2004).

\section{Orthostatic tolerance}

After HDBR, the time of OT in the control group was decreased to $50.9 \%$ of pre-HDBR values. The underlying mechanism of orthostatic intolerance might involve hypovolemia, muscle atrophy, alterations of hormone and metabolic systems, increases in venous dispensability, inability to elevate peripheral resistance adequately, and changed autonomic nervous activity during space-flight or HDBR (Pavy-Le Traon et al. 2007, Zuj et al. 2012), as well diverse capacity for vasoconstriction (Sundblad et al. 2014).

In present study, PV, cardiac systolic function and autonomic nervous activity were preserved, which might contribute to the preserved OT. AG-based moderate exercise training partially reduced the trend of decline in parasympathetic activity and increases in sympathetic activity due to HDBR. An underlying mechanism is can be postulated. During exercise the sympathetic nervous system had a primary role but withdrawal of parasympathetic activity is also important. The response of the sympathetic nervous to exercise is positively related to exercise intensity, the greater the intensity the greater the sympathetic activity. However, the neural system could adapt to exercise. Long-term aerobic exercise could result inhibition of sympathetic nervous and the enhancement of vagal nervous at rest and during same external workload as pre-training, which would slow down basic HR and improve HR reserve (Carter et al. 2003). The effect of exercise was the opposite to the effect of HDBR. Moreover, loss of muscle pumps might contribute to the onset of neural mediated syncope (Halliwill et al. 2014). In the present study, muscle strength in the lower body of subjects with a countermeasure might have been preserved, because exercise involves these muscle groups during cycle movement. When executing cycling ergometric exercise, subjects must finish a cycle by conquering resistance from the ergometer, which is beneficial to muscles in the lower limbs.

However, there was still about $22.5 \%$ decrease of OT in the CM group, although there is no significance. This is almost equal to the result of $30 \mathrm{~min}$ AG $(1 \mathrm{Gz}$ at mess cantor and $2 \mathrm{Gz}$ at foot level) exposure without exercise (Linnarsson et al. 2015). It seems that the individual who has higher aerobic power tend to reduce more values of OT. The higher exercise load may be suitable for the individual with higher aerobic power. Furthermore, the exercise load performed by subjects was suited for maintain aerobic power in the $1-\mathrm{Gz}$ normal environment, it is too mild to improve the LBNP-based orthostatic stress after HDBR. During microgravity or HDBR the human being need more intensive exercise stimulation to maintain physical fitness. According to the training principle of reversibility, when an athlete stops or reduces training for a period time, the improvements previously observed are reversed, i.e. detraining occurs. Therefore, the higher exercise intensity should be investigated in the future.

\section{Exercise capacity}

After HDBR, exercise endurance decreased by $3.5 \%$ in the control group, while was preserved in the $\mathrm{CM}$ group. $V \mathrm{O}_{2 \max }$ was decreased by $8.5 \%$, and AT was decreased by $8.2 \%$, respectively, in the control group. The $V \mathrm{O}_{2 \max }$ decreased a little more than the result (decreased by $5 \%$ in $V \mathrm{O}_{2 \max }$ ) of a 5-days HDBR (Linnarsson et al. 2015). But the result support each other, HDBR did reduce $V \mathrm{O}_{2 \max }$, which exceeded our expectation. We had supposed that the $V \mathrm{O}_{2 \max }$ should not change, or just changed a little, and the AT would reduce by the HDBR. However, the exercise endurance presented by the duration time of graded exercise was decreased only $3.5 \%$, which is less than the other two. During the grade exercise test, the part before the AT was dominated by aerobic metabolism. Therefore, these results suggested that the reduction of exercise capacity 
was more concerned with aerobic power though the general capacity was reduced by HDBR. The present countermeasure of artificial gravity combined with exercise preserved exercise capacity effectively. Even the aerobic power of some subjects trend to improve, which should be connected with the individuals physical fitness. All subjects were from ordinary college or graduate students without athlete experience. Maybe the exercise intensity is suited for them to improve aerobic power, while the exercise load is too mild to improve the LBNPbased orthostatic stress.

Decreased $V \mathrm{O}_{2 \max }$ is a consistent finding following HDBR and this effect is magnified when exercise testing is performed during the upright, rather than supine, posture (Convertino et al. 1982a). Therefore we choose upright posture to do exercise test. In a study on 20 days of HDBR, Katayama et al. (2004) applied two sessions of training, which was a combination of high/low G-load with low/high intensity exercise. One session was set at a $0.8-1.4 \mathrm{G}$ load at the heart level with constant exercise intensity $(60 \mathrm{~W})$. The second session began with a $0.3 \mathrm{G}$ load at the heart level with an interval exercise protocol $\left(40-80 \% V_{2} \mathrm{O}_{2 \max }\right)$. They found that $V \mathrm{O}_{2 \max }$ did not show a significant decrease after HDBR, which is consistent with the present study. The underlying mechanism of this finding might be related to two aspects. First, well-maintained cardiac function is possibly a critical factor for the preserved $V \mathrm{O}_{2 \max }$. In the present study, results from measurement of cardiac systolic function indicated that even 4 days of HDBR resulted in an apparent increase in PEP/LVET and a decrease in EF, showing a remarkable down-regulation of cardiac systolic function. The present countermeasure regimen that combination of $\mathrm{AG}(2 \mathrm{Gz}$ at foot level) and aerobic exercise (80-95\% AT) has effectively maintained cardiac systolic function. Even though lung function is thought to be another factor contributing to $V \mathrm{O}_{2 \max }$, our previous study showed that this physiological function was not affected by 4 days of HDBR (Guo et al. 2013). Second, exercised muscles in the limbs of subjects with AG-based cycling exercise might contribute to $V_{2}$ max . Greenleaf et al. (1989) confirmed that muscle exercise plays an important part in maintenance of aerobic power and muscle strength.

\section{Limitations}

The present study was carried out on HDBR for only 4 days, which was a major limitation. However, 4 days of HDBR induced some deconditioning of cardiovascular function, such as reduced PV, cardiac systolic function, and aerobic capability. Muscle atrophy and bone loss do not occur unless a longer duration of HDBR is performed. Furthermore, a single-treated matched group of aerobic cycling exercise or AG was absent. Such group would be helpful to ensure the effects of aerobic cycling exercise or AG on different physical deconditioning. And it would be important to adjust the AG does, exercise intensity, and the mode of the combined protocol in the future studies. And the AG load was set at the foot level in the present study, perhaps optimal setting should be according the Gz-load at the heart level or the center of mess. As mentioned above, a regulated training regimen based on the present study should be conducted in the future during long-term HDBR to evaluate the efficiency of AG-based exercise.

\section{Conclusion}

In summary, we investigated the potential beneficial effect of AG-based moderate exercise during 4 days of HDBR in humans. A regimen of $80-95 \%$ AT aerobic cycle ergometric exercise based on AG ( $2 \mathrm{Gz}$ at the foot level) successfully eliminates changes in OT, exercise capacity, cardiac systolic function, HRV, PV, and aldosterone concentration levels during 4 days of HDBR. Mechanisms of the improvements are not just cardiac-mediated, but also involve preserved blood volume and improved sympathetic responsiveness to orthostatic stress. The efficiency of an individualized high-impact aerobic plus anaerobic cycling exercise based on an AG training protocol should be further investigated during long-term simulated microgravity.

\section{Conflict of Interest}

There is no conflict of interest.

\section{Acknowledgements}

We thank the participants for their time and co-operation. We also thank Dr. Ming Jun Zhang, Department of Human Movement Studies, Xi'an Physical Education University, for technical assistance in exercise test. This study was supported by grants from the National Natural Science Foundation of China (81471817, 81301682), the Defense Medical Foundation of China (AWS12J003-01), Shaanxi Exercise Science Research Program and the Fundamental Research Funds for the Central Universities of China (7214518402). 


\section{References}

AUBERT AE, BECKERS F, VERHEYDEN B: Cardiovascular function and basics of physiology in microgravity. Acta Cardiol 60: 129-151, 2005.

BROWN TE, BEIGHTOL LA, KOH J, ECKBERG DL: Important influence of respiration on human R-R interval power spectra is largely ignored. $J$ Appl Physiol 75: 2310-2317, 1993.

BUCKEY JC, LANE LD, LEVINE BD, WATENPAUGH DE, WARIGHT SJ, MOORE WE, GAFFNEY FA, BLOMQVIST CG: Orthostatic intolerance after spaceflight. J Appl Physiol 81: 7-18, 1996.

CARTER JB, BANISTER EW, BLABER AP: Effect of endurance exercise on autonomic control of heart rate. Sports Med 33: 33-46, 2013.

COOKE WH, AMES JE IV, CROSSMAN AA, COX JF, KUUSELA TA, TAHVANAINEN KU, MOON LB, DRESCHER J, BAISCH FJ, MANO T, LEVINE BD, BLOMQVIST CG, ECKBERG DL: Nine months in space: effects on human autonomic cardiovascular regulation. J Appl Physiol 89: 1039-1045, 2000.

CONVERTINO VA, GOLDWATER DJ, SANDLER H: Effect of orthostatic stress on exercise performance after bedrest. Aviat Space Environ Med 53: 652-657, 1982a.

CONVERTINO VA, SANDLER H, WEBB P, ANNIS JF: Induced venous pooling and cardiorespiratory responses to exercise after bed rest. J Appl Physiol Respir Environ Exerc Physiol 52: 1343-1348, 1982 b.

CUSTAUD MA, DE SOUZA NETO EP, ABRY P, FLANDRIN P, MILLET C, DUVAREILLE M, FORTRAT J-O, GHARIB C: Orthostatic tolerance and spontaneous baroreflex sensitivity in men versus women after 7 days of head-down bed rest. Auton Neurosci 100: 66-76, 2002.

EVANS JM, STENGER MB, MOORE FB, HINGHOFER-SZALKAY H, ROSSLER A, PATWARDHAN AR, BROWN DR, ZIEGLER MG, KNAPP CF: Centrifuge training increases presyncopal orthostatic tolerance in ambulatory men. Aviat Space Environ Med 75: 850-858, 2004.

FRISTSCH-YELLE JM, CHARLES JB, JONES MM, BEIGHTOL LA, ECKBERG DL: Spaceflight alters autonomic regulation of arterial pressure in humans. $J$ Appl Physiol 77: 1776-1783, 1994.

GOSWAMI N, BRUNER M, XU D, BAREILLE M-P, BECK A, HINGHOFER-SZALKAY H, BLABER AP: Shortarm human centrifugation with $0.4 \mathrm{~g}$ at eye and $0.75 \mathrm{~g}$ at heart level provides similar cerebrovascular and cardiovascular responses to standing. Eur J Appl Physiol 115: 1569-1575, 2015.

GRRENLEAF JE, BERNAUER EM, ERTL AC, TROWBRIGHE TS, WADE CE: Work capacity during 30 days of bed rest with isotonic and isokinetic exercise training. J Appl Physiol 67: 1820-1826, 1989.

GRRENLEAF JE, CHOU JL, STAD NJ, LEFTHERIOTIS GP, ARNDT NF, JACKSON CG, SIMONSON SR, BARNES PR: Short-arm $(1.9 \mathrm{~m})+2.2 \mathrm{Gz}$ acceleration: isotonic exercise load-O2 uptake relationship. Aviat Space Environ Med 70: 1173-1182, 1999.

GUINET P, SCHNEIDER SM, MACIAS BR, WATENPAUGH DE, HUGHSON RL, LE TRAON AP, BANSARD J-Y, HARGENS AR: WISE-2005: effect of aerobic and resistive exercises on orthostatic tolerance during 60 days bed rest in women. Eur J Appl Physiol 106: 217-227, 2009.

GUO Y, GUO N, LIU C, WANG D, WANG J, SUN X, FAN S, WANG C, YANG C, ZHANG Y, LU D, YAO Y: Effect of artificial gravity with exercise training on lung function during head-down bed rest in humans. Clin Physiol Funct Imaging 33: 24-29, 2013.

HALLIWILL JR, SIECK DC, ROMERO SA, BUCK TM, ELY MR: Blood pressure regulation X: what happens when the muscle pump is lost? Post-exercise hypotension and syncope. Eur J Appl Physiol 114: 561-578, 2014.

HARGENS AR, BHATTACHARYA R, SCHNEIDER SM: Space physiology VI: exercise, artificial gravity, and countermeasure development for prolonged space flight. Eur J Appl Physiol 113: 2183-2192, 2013.

HINGHOFER-SZALKAY H, ROSSLER A, SCHARFETTER H, PLIZ K, LASZLO Z: Fluid volume changes and LBNP response after simulated weightlessness with varied oral sodium supply. J Gravit Physiol 9: P99-P100, 2002a.

HINGHOFER-SZALKAY HG, LASZLO Z, JEZOVA D, ROSSLER A, HADITSCH B, PILZ K, PASSATH H, SHARFETTER H: Bed rest immobilization with various oral sodium supply: plasma hormones and body fluids. Endocr Regul 36: 151-159, 2002b. 
HIRSCH JA, BISHOP B: Respiratory sinus arrhythmia in humans: how breathing pattern modulates heart rate. Am J Physiol 241: 620-629, 1981.

HUGHSON RL: Recent findings in cardiovascular physiology with space travel. Respir Physiol Neurobiol 169 (Suppl 1): S38-S41, 2009.

IWASAKI K, SASAKI T, HIRAYANAGI K, YAJIMA K: Usefulness of daily $+2 \mathrm{Gz}$ load as a countermeasure against physiological problems during weightlessness. Acta Astronaut 49: 227-235, 2001.

IWASAKI K, SHIOZAWA T, KAMIYA A, MICHIKAMI D, HIRAYANAGI K, YAJIMA K: Hypergravity exercise against bed rest induced changes in cardiac autonomic control. Eur J Appl Physiol 94: 285-291, 2005.

IWASAKI K, OGAWA Y, AOKI K, YANAGIDA R: Cerebral circulation during mild plus Gz hypergravity by short-arm human centrifuge. $J$ Appl Physiol 112: 266-271, 2012.

KATAYAMA K, SATO K, AKIMA H, ISHIDA K, TAKADA H, WATANABE Y, IWASE M, MIYAMURA M, IWASE S: Acceleration with exercise during head-down bed rest preserves upright exercise responses. Aviat Space Environ Med 75: 1029-1035, 2004.

LEE SM, MOORE AD, EVERETT ME, STENGER MB, PLATTS SH: Aerobic exercise deconditioning and countermeasures during bed rest. Aviat Space Environ Med 81: 52-63, 2010.

LEVINE BD, ZUCKERMAN JH, PAWELCZYK JA: Cardiac atrophy after bed-rest deconditioning: a nonneural mechanism for orthostatic intolerance. Circulation 96: 517-525, 1997.

LINNARSSON D, HUGHSON RL, FRASER KS, CLEMENT G, KARLSSON LL, MULDER E, PALOSKI WH, RITTWEGER J, WUYTS FL, ZANGE J: Effects of an artificial gravity countermeasure on orthostatic tolerance, blood volumes and aerobic power after short-term bed rest (BR-AG1). J Appl Physiol 118: 29-35, 2015.

MAILLET A, PAVY-LE TRAON A, ALLEVARD AM, SIGAUDO D, HUGHSON RL, GHARIB C, GAUQUELIN G: Hormone changes induced by 37.5-h head-down tilt (-6 degrees) in humans. Eur J Appl Physiol Occup Physiol 68: 497-503, 1994.

MAILLET A, GAUQUELIN G, GUNGA HC, FORTRAT JO, KIRSCH K, GUELL A, BIZOLLON CHA, GHARIB C: Blood volume regulating hormones response during two space related simulation protocols: four-week confinement and head-down bed-rest. Acta Astronaut 35: 547-552, 1995.

MOORE AD, LEE SMS, STENGER MB, PLATTS SH: Cardiovascular exercise in the U.S. space program: past, present and future. Acta Astronaut 66: 974-988, 2010.

MULDER ER, HORSTMAN AM, STEGEMAN DF, DE HAAN A, BELAVY DL, MIOKOVIC T, ARMBRECHT G, FELSENBERG D, GERRITS KH: Influence of vibration resistance training on knee extensor and plantar flexor size, strength, and contractile speed characteristics after 60 days of bed rest. $J$ Appl Physiol 107: 1789-1798, 2009.

MULDER E, CLEMENT G, LINNARSSON D, PALOSKI WH, WUYTS FP, ZANGE J, FRINGS-MEUTHEN P, JOHANNES B, SHUSHAKOV V, GRUNEWALD M, MAASSEN N, BUEHLMEIER J, RITTWEGER J: Musculoskeletal effects of 5 days of bed rest with and without locomotion replacement training. Eur $J$ Appl Physiol 115: 727-738, 2015.

NORSK P, BONDE-PETERSEN F, WARBERG J: Blood pressure regulation IV: adaptive responses to weightlessness. Eur J Appl Physiol 114: 481-497, 2014.

PAGANI M, LOMBARDI F, GUZZETTI S, RIMOLDI O, FURLAN R, PIZZINELLI P, SANDRONE G, MALFATTO G, DELL'ORTO S, PICCALUGA E: Power spectral analysis of heart rate and arterial pressure variabilities as a marker of sympatho-vagal interaction in man and conscious dog. Circ Res 59: 178-193, 1986.

PAVY-LE TRAON A, HEER M, NARICI MV, RITTWEGER J, VERNIKOS J: From space to Earth: advances in human physiology from 20 years of bed rest studies (1986-2006). Eur J Appl Physiol 101: 143-194, 2007.

RITTWEGER J, BAREILLE M-P, CLEMENT G, LINNARSSON D, PALOSKI WH, WUYTS F, ZANGE J, ANGERER O: Short-arm centrifugation as a partially effective musculoskeletal countermeasure during 5-day head-down tilt bed rest - results from the BRAG1 study. Eur J Appl Physiol 115: 1233-1244, 2015.

SCHMEDTJE JF JR, LIU WL, TAYLOR AA: Cardiovascular deconditioning through head-down tilt bed rest increases blood pressure variability and plasma renin activity. Aviat Space Environ Med 67: 539-546, 1996. 
SMITH SM, ZWART SR, HEER M, LEE SM, BAECKER N, MEUCHE S, MACIAS BR, SHACKELFORD LC, SCHNEIDER S, HARGENS AR: WISE-2005: supine treadmill exercise within lower body negative pressure and flywheel resistive exercise as a countermeasure to bed rest-induced bone loss in women during 60-day simulated microgravity. Bone 42: 572-581, 2008.

STENGER MB, EVANS JM, KNAPP CF, LEE SM, PHILLIPS TR, PEREZ SA, MOORE AD JR, PALOSKI WH, PLATTS SH: Artificial gravity training reduces bed rest-induced cardiovascular deconditioning. Eur J Appl Physiol 112: 605-616, 2012.

STENGER MB, LEE SMC, RIBEIRO LC, PHILLIPS TR, PLOUTZ-SNYDER RJ, WILLIG MC, WESTBY CM, PLATTS SH: Gradient compression garments protect against orthostatic intolerance during recovery from bed rest. Eur J Appl Physiol 114: 597-608, 2014.

SUNDBLAD P, KOLEGARD R, EIKEN O: G tolerance and the vasoconstrictor reserve. Eur J Appl Physiol 114: 2521-2528, 2014.

TRUDEL G, COLETTA E, CAMERON I, BELAVY DL, LECOMPTE M, ARMBRECHT G, FELSENBERG D, UHTHOFF HK: Resistive exercises, with or without whole body vibration, prevent vertebral marrow fat accumulation during 60 days of head-down tilt bed rest in men. $J$ Appl Physiol 112: 1824-1831, 2012.

VAN BEAUMONT W: Evaluation of hemoconcentration from hematocrit measurements. J Appl Physiol 32: 712-713, 1972.

VERNIKOS J, LUDWIG DA, ERTL AC, WADE CE, KEIL L, O'HARA D: Effect of standing or walking on physiological changes induced by head down bed rest: implications for spaceflight. Aviat Space Environ Med 67: 1069-1079, 1996.

WANG YC, YANG CB, WU YH, GAO Y, LU DY, SHI F, WEI XM, SUN XQ: Artificial gravity with ergometric exercise as a countermeasure against cardiovascular deconditioning during 4 days of head-down bed rest in humans. Eur J Appl Physiol 111: 2315-2325, 2011.

WATENPAUGH DE: Fluid volume control during short-term space flight and implications for human performance. J Exp Biol 204: 3209-3215, 2011.

WATERS WW, ZIEGLER MG, MECK JV: Postspaceflight orthostatic hypotension occurs mostly in women and is predicted by low vascular resistance. J Appl Physiol 92: 586-594, 2002.

YANG CB, ZHANG S, ZHANG Y, WANG B, YAO YJ, WANG YC, WU YH, LIANG WB, SUN XQ: Combined short-arm centrifuge and aerobic exercise training improves cardiovascular function and physical working capacity in humans. Med Sci Monit 16: 575-583, 2010.

YANG CB, WANG YC, GAO Y, GENG J, WU YH, ZHANG Y, SHI F, SUN XQ: Artificial gravity with ergometric exercise preserves the cardiac, but not cerebrovascular, functions during 4 days of head-down bed rest. Cytokine 56: 648-655, 2011.

YAO YJ, ZHU YS, YANG CB, ZHOU XD, SUN XQ: Artificial gravity with ergometric exercise can prevent enhancement of popliteal vein compliance due to 4-day head-down bed rest. Eur J Appl Physiol 112: 1295-1305, 2012.

ZUJ KA, ARBEILLE P, SHOEMAKE JK, BLABER AP, GREAVES DK, XU D, HUGHSON RL: Impaired cerebrovascular autoregulation and reduced $\mathrm{CO}(2)$ reactivity after long duration spaceflight. Am $J$ Physiol Heart Circ Physiol 302: H2592-H2598, 2012. 